\title{
Nurse practitioner management of acute in-hours home visit or assessment requests: a pilot study
}

\author{
Martin Edwards, Carol Bobb and Susan I Robinson
}

\begin{abstract}
\section{Background}

GPs often perceive home-visit requests as a timeconsuming aspect of general practice. The new general medical services contract provides for practices to be relieved of responsibility for homevisits, although there is no model for the transfer of care. One such model could be to employ nurse practitioners to manage such requests. Nurse practitioners can effectively substitute for GPs in managing same-day in-hours emergency care in the surgery, but their role in managing all such requests, including those requiring home visits, has not been assessed.
\end{abstract}

Aim

To explore the feasibility and clinical management outcomes of nurse practitioner management of sameday care requests, including those requiring home visits, to inform a proposed randomised controlled trial.

\section{Design of study}

Non-randomised comparative trial.

\section{Setting}

One large general practice (14 600 patients) in south London.

\section{Method}

Nurse practitioner assessment and management of all same-day care requests for 2 days per week was compared with normal GP management on another 2 days, over a 6-month period. Clinical management outcome data were collected from patient records and from data-collection forms completed by a nurse practitioner and GPs. Patient and staff satisfaction was assessed by questionnaire.

\section{Results}

The nurse practitioner was more likely than GPs to assess patients in person, less likely to give advice alone, and more likely to issue a prescription. There was no significant difference between the nurse practitioner and GPs regarding any other clinical management outcomes or patient satisfaction; however, the response rate of the patient satisfaction questionnaire in this pilot study was poor.

\section{Conclusion}

Nurse practitioner management of acute in-hours care requests, including home visits, appears feasible in practice and merits further assessment.

\section{Keywords}

house calls; nurse practitioners; primary health care.

\section{INTRODUCTION}

Whatever their out-of-hours arrangements may be, GPs in Britain are generally required to manage demands for immediate advice or treatment that occur within normal working hours. For patients presenting as acute 'same-day' or emergency cases to a GP surgery, nurse practitioners appear acceptable to them as a first or only point of contact. ${ }^{1,2}$ Randomised controlled trials suggest that nurses who are solely responsible for the diagnosis and management of these patients achieve equivalent clinical outcomes to GPs, ${ }^{3,4}$ with possibly higher levels of patient satisfaction, although their consultations are longer. ${ }^{5}$ To date, none of these studies has examined the feasibility of nurse practitioners extending their activity to home visits of patients who are acutely ill. Substituting a nurse practitioner to assess these requests, and to perform home visits if necessary, could potentially free up a substantial amount of GP time.

The suggestion that nurse practitioners could manage home-visit requests is not new; Jiwa et al, in an analysis of primary care out-of-hours visits, proposed that the nurse's role could realistically be extended from telephone triage to visiting and managing patients. ${ }^{5}$ Few providers have employed nurse practitioners in this way, although in one study emergency care practitioners (emergency medical

M Edwards, MD, MRCGP, GP, Jenner Practice, Forest Hill, London. C Bobb, BSc (Hons) NP Dip.RCN, nurse practitioner, Kingston University Health Centre, Fairhill Medical Practice, Kingston-Upon-Thames. SI Robinson, $\mathrm{PhD}$, primary care research fellow, Lewisham Research Unit, Cantilever House, London.

Address for correspondence

Dr Martin Edwards, Jenner Practice, 201 Stanstead Road, Forest Hill, London, SE23 1HU.

E-mail: mail9151@martinedwards.me.uk

Submitted: 14 February 2008; Editor's response: 9 April 2008; final acceptance: 1 July 2008.

(c)British Journal of General Practice.

This article was originally online first. Cite this article as: Br J Gen Pract 2009; 59: 7-11. Advance online publication. DOI: $10.3399 /$ bjgp09X394798 


\section{How this fits in}

Requests for same-day assessment, particularly at a patient's home, are perceived as a time-consuming aspect of general practice. This study suggests that it is feasible for nurse practitioners to manage such requests, including undertaking home visits, if appropriate. Nurse practitioner management differed from GP care only in terms of nurse practitioners giving a higher proportion of face-to-face contact and having a higher prescription rate.

technicians or paramedics who have undergone a further diploma course) appeared acceptable to patients as providers of out-of-hours emergency home assessment. ${ }^{6}$ Studies involving a wider role of nurses in managing acute illness at home in selected groups of patients, such as older people ${ }^{7}$ or patients recently discharged from hospital, ${ }^{8}$ also indicate that nurses are competent to adopt this role, and appear to value and enjoy it. ${ }^{8}$ However, nurse practitioner management of home-visit requests to GP practices within normal working hours (defined in the new general medical services [GMS] contract as 08:00-18:30) has not formed the subject of a study so far.

The new GMS contract provides for primary care trusts to relieve GPs of the responsibility for in-hours home visits when this is considered 'feasible and desirable'. ${ }^{9}$ However, there is little reported experience of such transfer of responsibility, and little information regarding potential models of care or patients' views of such a change in working. This study examined the feasibility and acceptability of substituting nurse practitioners for GPs to manage in-hours acute sameday assessment requests, including home-visit requests, to inform service development and the design of a future randomised controlled trial.

\section{METHOD}

This study was conducted in one large group practice (14 600 patients) in south London. Data collection took place over 6 months from October 2006 to March 2007. The practice's usual procedure is to allocate one GP as a duty doctor for the day, who is solely responsible for responding to patient requests for inhours urgent or same-day advice or assessment, as well as undertaking some routine administrative tasks. The duty doctor is not allocated any routine prebooked patients. During the operation of the study, these responsibilities were effectively adopted by a single nurse practitioner who was responsible, on 2 working days each week for 6 months, for responding to in-hours patient requests for immediate assessment, including acute home-visit requests. On the remaining 3 days of the week, such requests were managed by a duty GP, according to the practice's usual procedure. Mondays were excluded from data collection as this day generates a higher workload than the remaining weekdays, and patients tend to have different presenting symptoms and demography.

Nurse practitioner management (on Tuesdays and Wednesdays) was compared with normal GP management (on Thursdays and Fridays). Patients who had previously been enrolled in the study remained eligible for recruitment again, provided that their new contact did not relate to the same clinical care episode. Contacts that related to a previous episode were coded as a follow-up for that episode.

Adult patients suffering acute minor illness, acute injury, chronic disease (excluding routine management), or an acute exacerbation of chronic disease were included in the study. Those who were excluded were patients under 18 years; those with a presenting complaint of mental illness involving psychosis, suicidal ideation, a risk of harm to self or others, or requiring assessment for detention under a section of the Mental Health Act 1983; those requesting confirmation of death; or those with illness or complications due to pregnancy. These patients were managed by the duty GP in the normal way. These exclusions were deemed, by the study nursing advisers and the nurse practitioner, to fall outside the usual expected competence of a nurse practitioner.

The nurse practitioner was a qualified independent prescriber with 12 years' experience of working in general practice. Upon initial contact (generally by telephone) with a patient who was eligible, the nurse practitioner's available options were to:

- offer telephone advice alone;

- request that the patient attend surgery for nurse or doctor assessment;

- visit the patient at home;

- seek advice from a GP regarding management; or

- deem the problem outside their area of clinical expertise and refer it to a GP.

The nature and outcome of each patient contact was recorded by the GP or nurse practitioner on a datacollection form and supplemented by researchers' scrutiny of the patient's computerised medical record. The nature of each contact (telephone, surgery, home) was recorded, together with the Read code classification of the presenting complaint, whether a physical examination was performed, and the clinical management of the episode.

Patients and/or their carers were asked to complete a questionnaire regarding their experience of the care they had received and were given a stamped addressed envelope for their reply. The questionnaire was either left with the patient by the GP or nurse practitioner at the time of consultation, to be completed later and posted back, or was posted to the patient within 7 days of the initial contact. It 
incorporated a validated instrument to assess satisfaction with the consultation experience (the Picker Institute's GP Questionnaire), ${ }^{10}$ together with measures of patient enablement, continuity, and practitioner empathy (using the Consultation Quality Index). ${ }^{11}$ Patients whose completed questionnaires had not been received within 2 weeks were sent a second questionnaire.

At the end of the study, practice staff (GPs, practice nurses, district nurses, and administrative staff) were asked to indicate how well they felt that the new arrangement had worked, by marking a linear scale from 'didn't work at all' to 'worked perfectly'. They were also invited to describe their experiences of working with the nurse practitioner.

SPSS statistical analysis software (version 14.0) was used to compare the nature and outcome of GP and nurse practitioner consultations $\left(\chi^{2}\right)$ and patient questionnaire scores (analysis of variance.) As a nonrandomised pilot study with naturally occurring comparison groups, this investigation was not intended to demonstrate subtle differences between GP and nurse practitioner care, but rather to establish the feasibility and acceptability of the care model and methodology in order to inform a prospective, larger randomised controlled trial.

Patients were not asked to consent to nurse practitioner management. Lewisham ethics committee considered that such management fell within the normal range of services offered by the practice and that separate consent was unnecessary. Any patients who objected to nurse practitioner care would be offered normal care from a GP. Patients were asked to consent to scrutiny of their records by the researchers; they were told their records would be examined inpractice but that no patient-identifiable data would be extracted or retained.

\section{RESULTS}

A total of 568 contacts were analysed from 455 patients: 311 contacts were with a GP and 257 with the nurse practitioner. Twice as many contacts involved female patients as males; $22 \%$ of contacts were with patients $>80$ years of age.

There was no significant difference between nurse practitioner and GP consultations regarding patient age or sex. The nurse practitioner was significantly more likely than a GP to issue a prescription (56.9\% versus $43.1 \%, P<0.001)$ and to assess patients in person (58.4\% versus $42.4 \%, P<0.001$ ); and the nurse practitioner was less likely to manage contacts by advice alone (42.9\% versus $64.5 \%, P<0.001$; Table 1 ). The nurse practitioner sought advice from a GP in $8.6 \%$ of cases and asked a GP to see her patient in $0.8 \%$ of cases. There was no significant difference between nurse practitioner and GP contacts regarding rates of home visiting, patient admission to hospital, referral to outpatients, or requests for further investigation (blood tests, urine tests, or X-ray).

Patient questionnaire data revealed no significant difference in any of the dimensions (empathy, patient enablement, communication, or prescribing management) between GP and nurse practitioner management. However, the proportion of questionnaires returned $(21.5 \%$ overall, or 122 returned of 568 questionnaires issued) was too low to enable firm conclusions to be drawn. There was a significant difference in the response rate of patients, depending on whether they had consulted with a GP or nurse practitioner; 49 (15.75\%) GP questionnaires were returned compared with 73 (28.4\%) nurse practitioner questionnaires $(P<0.001)$. Older participants were more likely to return their questionnaires: $25 \%$ of those aged $>80$ years and $27 \%$ of all those aged $>50$ years returned theirs, compared with $15 \%$ of those aged $<50$ years.

Twenty-nine questionnaires were distributed to practice staff, of which only 16 questionnaires were returned and indicated a score on the linear scale. Staff who did not score the scale reported they had experienced insufficient contact with the nurse practitioner to do so. Half of the responders $(n=8)$ indicated that the arrangement had worked well, of whom five indicated that it had worked perfectly. However, the remainder responded that the arrangement had worked less well, with common concerns including insufficient integration of the nurse practitioner at the beginning of the study, inadequate

Table 1. Questionnaire results $(n=568)$.

\begin{tabular}{|c|c|c|c|c|}
\hline & $\begin{array}{c}\text { Nurse } \\
\text { practitioners }\end{array}$ & GPs & & \\
\hline & $n(\%)$ & $n(\%)$ & OR $(95 \% \mathrm{Cl})$ & $P$-value \\
\hline Managed by telephone alone & $107(41.6)$ & $179(57.6)$ & 0.77 (0.66 to 0.89$)$ & $<0.001$ \\
\hline Managed by advice alone & 109 (42.9) & $202(64.5)$ & 0.62 (0.52 to 0.75$)$ & $<0.001$ \\
\hline Total face-to-face contacts & $150(58.4)$ & $132(42.4)$ & 1.30 (1.12 to 1.52$)$ & 0.001 \\
\hline Home visit performed & 39 (15.2) & $42(13.5)$ & 1.06 (0.85 to 1.32$)$ & 0.610 \\
\hline Seen in surgery & $111(43.2)$ & $90(28.9)$ & 1.32 (1.11 to 1.57$)$ & 0.001 \\
\hline Prescription issued & $178(56.9)$ & $135(43.1)$ & 1.36 (1.13 to 1.64$)$ & 0.001 \\
\hline Admission to hospital & $6(2.4)$ & $12(3.8)$ & 0.74 (0.38 to 1.43 ) & 0.320 \\
\hline \multirow{2}{*}{$\begin{array}{l}\text { Patient referred for opinion, } \\
\text { therapy, or further investigation }\end{array}$} & $33(12.9)$ & $51(16.3)$ & 0.89 (0.74 to 1.08$)$ & 0.260 \\
\hline & Mean (SD) & Mean (SD) & & \\
\hline CARE score, range $10-50$ & 40 (9.62) & $36.4(9.92)$ & - & 0.054 \\
\hline PEI score, range 0-12 & $4.0(3.56)$ & $3.5(3.82)$ & - & 0.380 \\
\hline $\begin{array}{l}\text { Picker communication score, }{ }^{10} \\
\text { range } 0-600\end{array}$ & $549(93.9)$ & $520(122.7)$ & - & 0.145 \\
\hline $\begin{array}{l}\text { Picker medication score, }{ }^{10} \\
\text { range } 0-400\end{array}$ & $295(113.8)$ & 305 (123.3) & - & 0.712 \\
\hline
\end{tabular}

$C A R E=$ Consultation and Relational Empathy. ${ }^{11} \mathrm{OR}=$ odds ratio $P E I=$ Patient Enablement Instrument. ${ }^{11}$ 
staff preparation for the initial learning curve as the practice adapted to a new way of working, and, from some GPs, residual anxiety that the nurse practitioner was insufficiently qualified to assess complex medical issues.

\section{DISCUSSION}

\section{Summary of main findings}

This pilot study suggests that nurse practitioner assessment of same-day general practice care requests, including home-visit requests and undertaking the visit where necessary, appears feasible in practice. The nurse practitioner was more likely to assess patients face-to-face, and to prescribe; no other differences were observed in clinical management outcomes. Although no significant difference was observed in patient satisfaction ratings, the questionnaire response rate was too low to permit firm conclusions in this regard. Nevertheless, no substantial barriers were observed to this method of working, and the findings will help to inform the design of a randomised controlled trial.

\section{Strengths and limitations of the study}

This is an account of one nurse practitioner working in one practice with a particular method of accommodating same-day care requests, and the findings might not be generalisable to other practices with different patient populations and ways of working. The activity of one nurse practitioner was compared with the aggregated activity of seven GPs, who are likely to show variability among their own management decisions using telephone assessment. ${ }^{12}$ Therefore, the study findings cannot be extrapolated beyond the suggestion that no substantial obstacles to GP substitution by a nurse practitioner were demonstrated.

More patient contacts were recorded by GPs than the nurse practitioner (311 versus 257), due to the inclusion of data from days when the nurse practitioner was absent and her duties were covered by a GP. Employment of a pool of nurse practitioners, and randomisation of patients to nurse practitioner or GP care, should address this in a future larger study.

This pilot study did not examine consultation duration or relative costs of GP and nurse practitioner care. A future larger study would require sufficient resources to compare these parameters and also to allow for independent assessment of patients' clinical management and clinical outcome data.

The response rate of patient questionnaires was poor $(21.5 \%)$; the reason for this is not clear and, although age and frailty may have been a factor $(38 \%$ of participants were aged $>70$ years and $22 \%$ were $>80$ years), older participants actually proved to be better responders. Resources did not permit extensive follow-up of non-responders, other than sending them a further copy of the questionnaire. A telephone reminder, or the opportunity to complete the questionnaire over the telephone, might be helpful in a future study. Younger participants were poorer responders; it might be appropriate to seek their cooperation more vigorously by using additional techniques such as sending reminders via email or SMS. A larger study might also be able to finance a small reward for completing the questionnaire.

Significantly more questionnaires were returned by patients who saw the nurse practitioner as opposed to the GP, apparently reflecting the finding that the nurse practitioner was more likely to hand questionnaires to patients in person rather than relying on posting them after the contact. Although the tendency of the nurse practitioner to hand over questionnaires in person could conceivably bias her responders towards recording a positive score, the questionnaires were completed later and posted, not completed while the nurse practitioner was present. It appears, however, that the policy of handing over questionnaires in person would be a useful technique to implement in a future trial.

\section{Comparison with existing literature}

Although the low questionnaire response rate compromises the validity of the study, there was no indication of patient dissatisfaction with nurse practitioner management. Studies of nurse practitioners seeing same-day patients in practice premises similarly suggest high patient satisfaction scores, generally in excess of those achieved by GPs. ${ }^{1,3-4}$

These studies did not observe any increase in prescribing from nurse practitioners. The increased prescription rate seen in the present study might reflect the individual practitioner involved, or the fact that the clinical and demographic characteristics of the patients she was seeing in their homes differed from patients studied in a surgery.

\section{Implications for future research}

Attitudes of practice staff to the nurse practitioner's role were mixed and uncertainties largely focused on the initial few weeks while practitioners and staff adapted to new working arrangements. Careful integration of nurse practitioner personnel, together with an explanation of their role, will be an important prerequisite for a future study.

Patient questionnaire response rates would need to improve in a future study. The entire questionnaire could be routinely administered face-to-face by a researcher; this would be a more costly measure but would be likely to maximise response rates. Effective management of the response rate in a larger trial will be important if the findings are not to be compromised. 
Overall, extending nurse practitioner management to include in-hours home visits potentially offers a practical and feasible means of freeing up GP time for surgery work, while simultaneously removing a source of occupational GP stress. This pilot investigation merits further study in a larger randomised controlled trial, which should also include economic and time analyses as well as clinical outcome data.

\section{Funding body}

This study was supported by a Research Priority Scheme grant (grant no. RPS0601) from Southwark Research Support Unit (now the Research and Development Centre)

\section{Ethical approval}

Ethical approval was provided by Lewisham Research Ethics Committee (06/Q0701/30)

\section{Competing interests}

The authors have stated that there are none

\section{Acknowledgements}

Thanks to Picker Institute Europe, Oxford, UK for permission to adopt the GP Questionnaire, and to Drs Mercer, Howie, et al, for the use of CQI-2. Thanks to study management group members Jeanette Garforth and Julie Dennison for helping the smooth running of this study, and to Professor Alison While for advice on study design. Thanks to Lewisham PCT for acting as sponsor to this trial and to STarNet for initial, and Lewisham Research Unit for subsequent, academic support. Many thanks to the staff and patients of the Jenner Practice for their participation in this study

\section{Discuss this article}

Contribute and read comments about this article on the Discussion Forum: http://www.rcgp.org.uk/bjgp-discuss

\section{REFERENCES}

1. Myers PC, Lenci B, Sheldon MG. A nurse practitioner as the first point of contact for urgent medical problems in a general practice setting. Fam Pract 1997; 14(6): 492-497.

2. Reveley $\mathrm{S}$. The role of the triage nurse practitioner in general medical practice: an analysis of the role. J Adv Nurs 1998; 28(3): 584-591.

3. Kinnersley P, Anderson E, Parry K, et al. Randomised controlled trial of nurse practitioner versus general practitioner care for patients requesting 'same day' consultations in primary care. BMJ 2000; 320(7241): 1043-1048.

4. Shum C, Humphreys A, Wheeler D, et al. Nurse management of patients with minor illnesses in general practice: multicentre, randomised controlled trial. BMJ 2000; 320(7241): 1038-1043.

5. Jiwa M, Bakewell J, Foster S, Gerrish K. Primary care visits out-ofhours. The case for skill mix? J Community Nurs 2001; 15(11): 4-6.

6. Halter M, Marlow T, Mohammed D, Ellison GT. A patient survey of out-of-hours care provided by Emergency Care Practitioners. BMC Emerg Med 2007; 7: 4

7. Nicolaides-Bouman A, van Rossum E, Kempen GI, Knipschild P. Effects of home visits by home nurses to elderly people with health problems: design of a randomised clinical trial in the Netherlands. BMC Health Serv Res 2004; 4(1): 35.

8. Ecklund S. Increasing nurse satisfaction: providing home visit opportunities. Recruit Retent Rep 1993; 6(12): 3-7.

9. Department of Health. Investing in general practice: the new general medical services contract. London: Department of Health, 2003.

10. Picker Institute. GP questionnaire. http://www.pickereurope.org/page.php?id=65 (accessed 19 July 2008).

11. Mercer SW, Howie JG. CQI-2 - a new measure of holistic interpersonal care in primary care consultations. Br J Gen Pract 2006; 56(525): 262-268.

12. Moll van Charante EP, ter Riet G, Drost S, et al. Nurse telephone triage in out-of-hours GP practice: determinants of independent advice and return consultation. BMC Fam Pract 2006; 7: 74. 